\title{
Non-Flow Through Fuel Cell Power Module Demonstration on the Scarab Rover
}

lan Jakupca, Monica Guzik, William R. Bennett NASA Glenn Research Center

Lawrence Edwards

Vantage Partners, LLC

July 10, 2017 


\section{Overview}

- Demonstration Scope

- Fundamental Technologies

- Advanced Product Water Removal (APWR) Non-Flow Through (NFT) Reactant Management

- Quad-Cell Voltage Monitoring Board (QCVMB)

- Dust Tolerant Automated Umbilical (DTAU)

- Power Module

- Rover

- Integrated Vehicle

- Demonstration

- Results

- Questions?

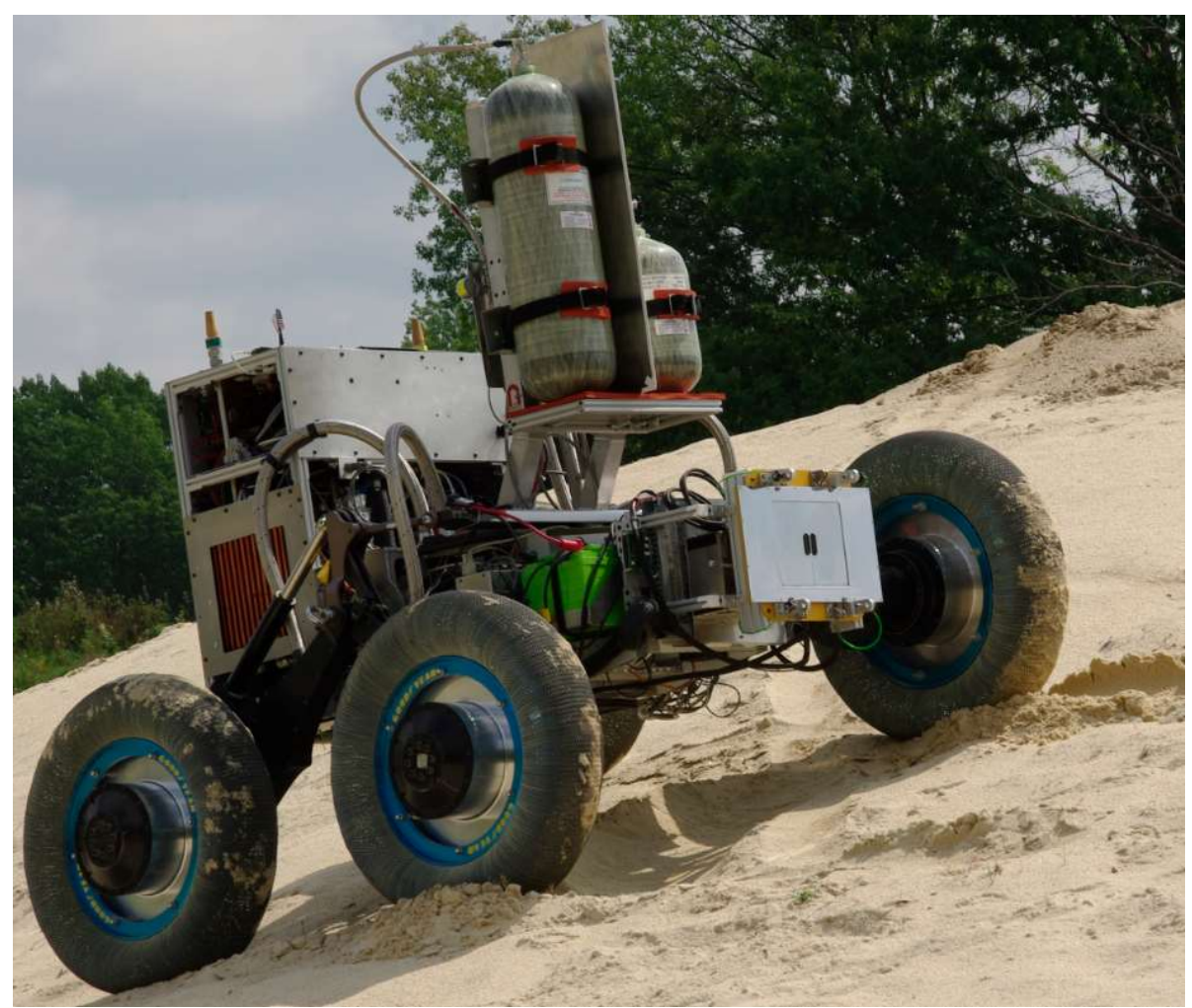




\section{Demonstration Scope}

- Provide tangible evidence illustrating that the Advanced Product Water Removal (APWR) Non-Flow Through (NFT) Reactant Management fuel cell technology has matured to TRL 5

- Flight-qualified $\mathrm{H}_{2} / \mathrm{O}_{2}$ fuel cell hardware is not currently available

- The most recent flight-qualified hardware was de-commissioned at the end of the Space Shuttle program

- Package APWR NFT fuel cell stack into a modular power system

- Design to allow for implementation onto multiple platforms without modifications

- Validate packaged fuel cell stack performance equivalent to laboratory performance

- Power a mobile platform using modular power system

- Validate full vehicle operational capability when powered by modular power system

- Validate that the fuel cell stack and modular power system are unaffected by disturbances (mechanical, electrical, thermal, etc.) imposed by the vehicle during mobile operations 


\section{Non-Flow-Through (NFT) Fuel Cell Technology}

- Advanced Product Water Removal (APWR) water management technology achieves Non-Flow Through (NFT) reactant control

- Surface tension and capillary forces passively "wick" water from each cell to a low-pressure water cavity

- Eliminates need for external water removal (reduces mass, increases reliability)

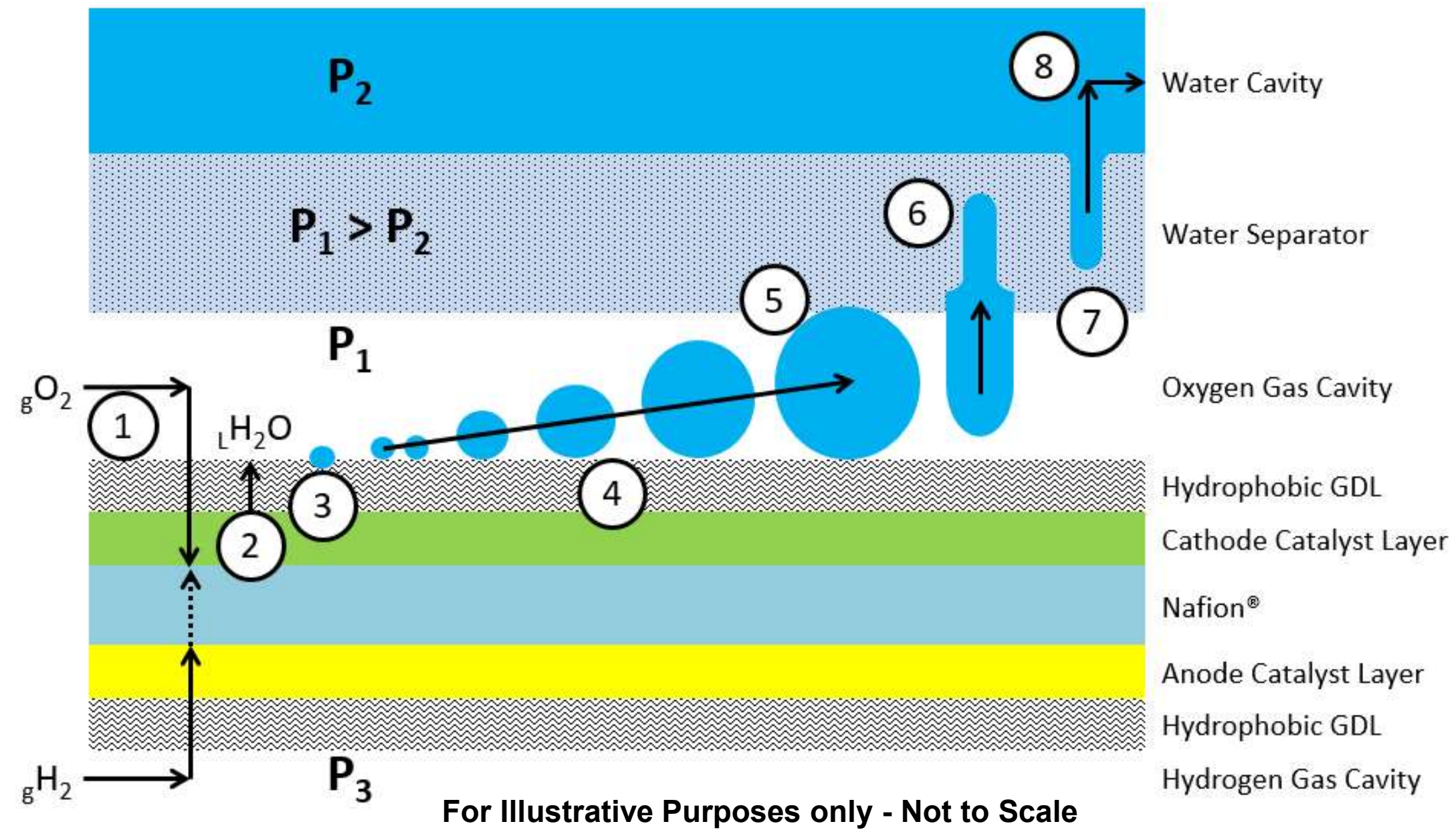




\section{Quad-Cell Voltage Monitoring Board (QCVMB)}

- PC-104 Industry Standard Module size (3.6" x 3.8")

- 48 Individual Cell Voltages $\left(0-2.5 V_{D C}\right)$

- 12 bit ADC enables $\pm 10 \mathrm{mV}$ cell voltage accuracy

- $33 \mathrm{~Hz}$ measurement rate (each cell voltage)

- 0 and $1 \mathrm{~V}_{\mathrm{DC}}$ reference voltages for in situ real-time calibration checks

- $600 \mathrm{~V}_{\mathrm{DC}}$ Isolation between signals and board

- On-board MCU 4 DIO; USB, UART, I²C communication

- Programmable in-situ fault detection/health monitoring tables

- 16 Single Ended (or 8 differential ) 0-1 $V_{D C}$ range inputs with 12 bit ADC; $33 \mathrm{~Hz}$ measurement rate

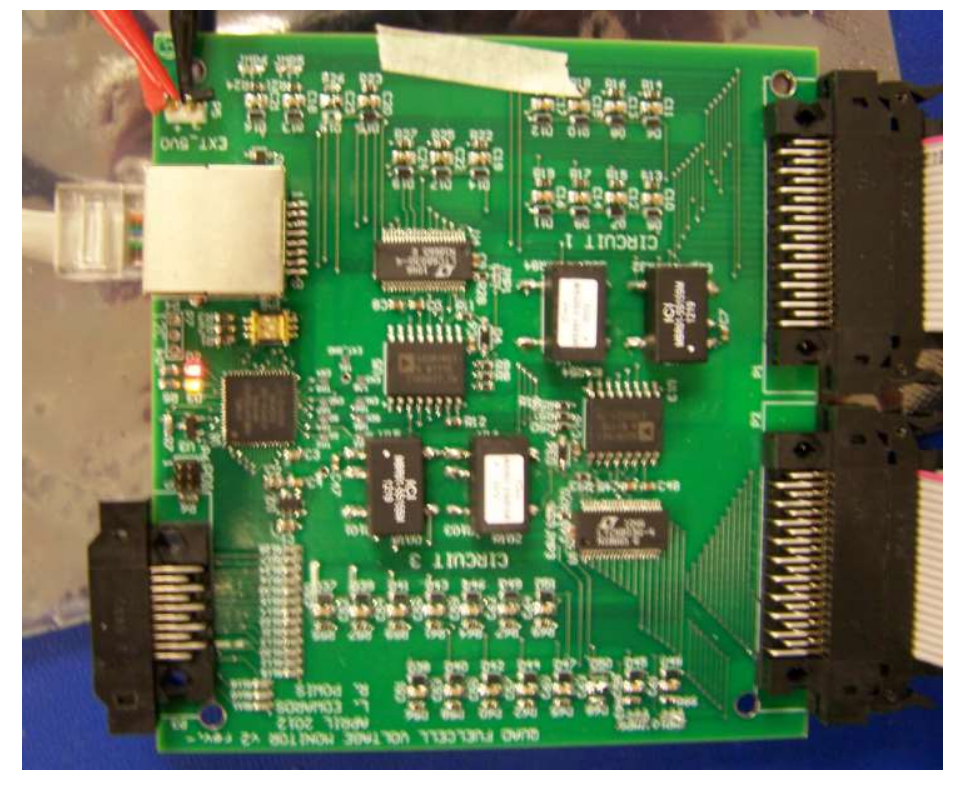

- 6 LED status indicators

- 1.5 watt consumption on a $5 \mathrm{~V}_{\mathrm{DC}}$ bus

- Up to 8 boards can be interfaced simultaneously to the control processor (384 cells) 


\section{Dust Tolerant Automated Umbilical (DTAU)}

- Prototype hardware with COTS quick connects (QCs) in a dust-resistant enclosure

- Halves are manually latched and a motorized drive brings the QCs together

- Encoder detects when QCs are fully mated

- Box and QC compartment are purged with GN2

- Procedures to confirm that QCs are mated and leak-proof

- $\mathrm{H} 2$ detector monitors purge gas

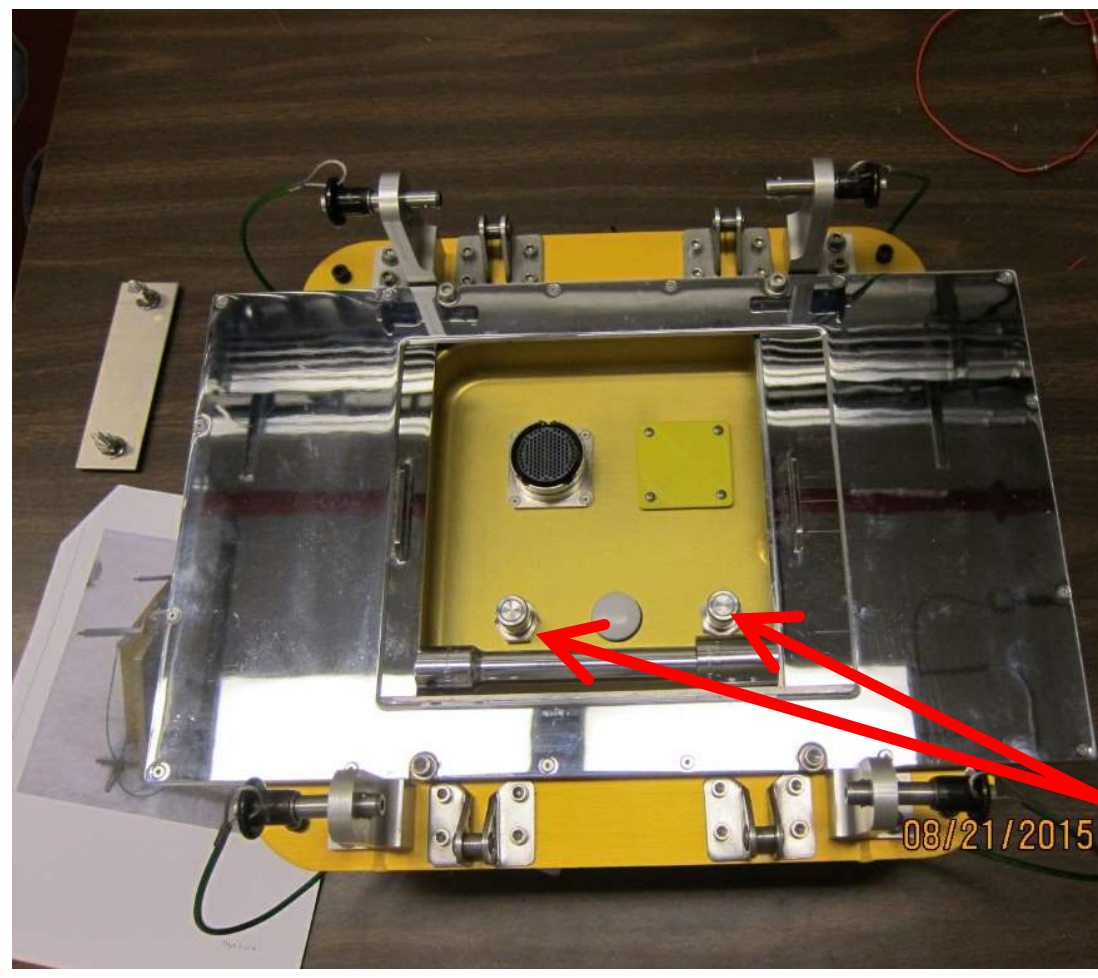

Passive half mounted on Scarab

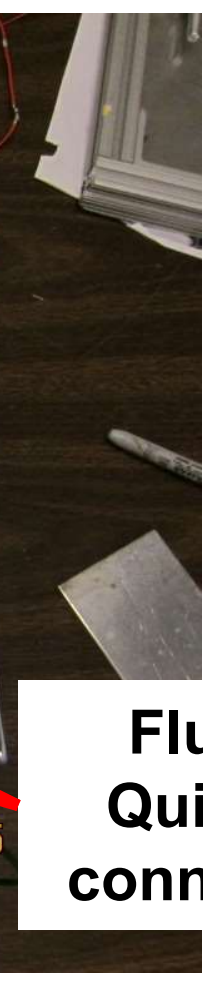

Fluid Quickconnects

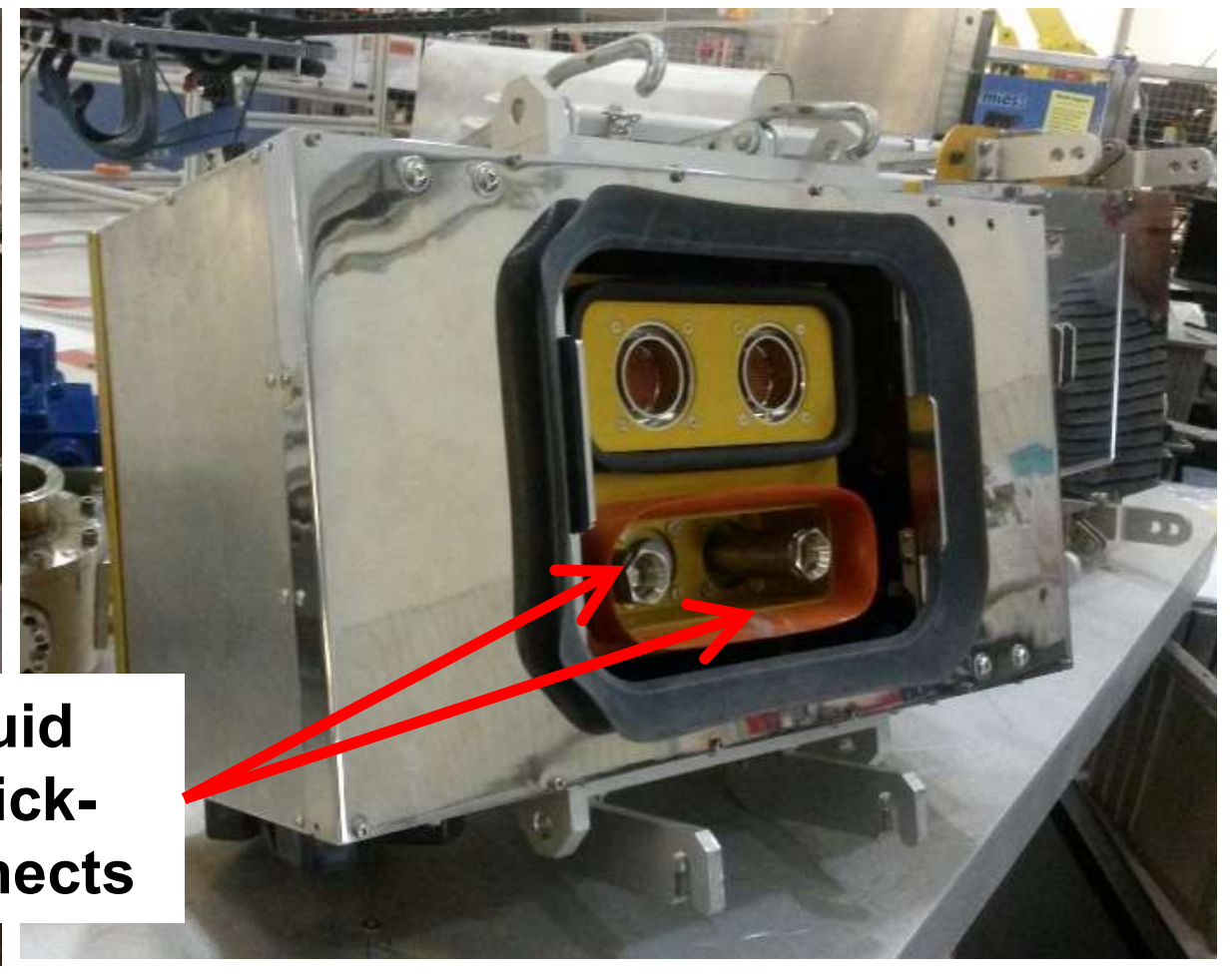

Active half manually latched to passive half for fluid transfer 


\section{Overview of a Fuel Cell Power System}

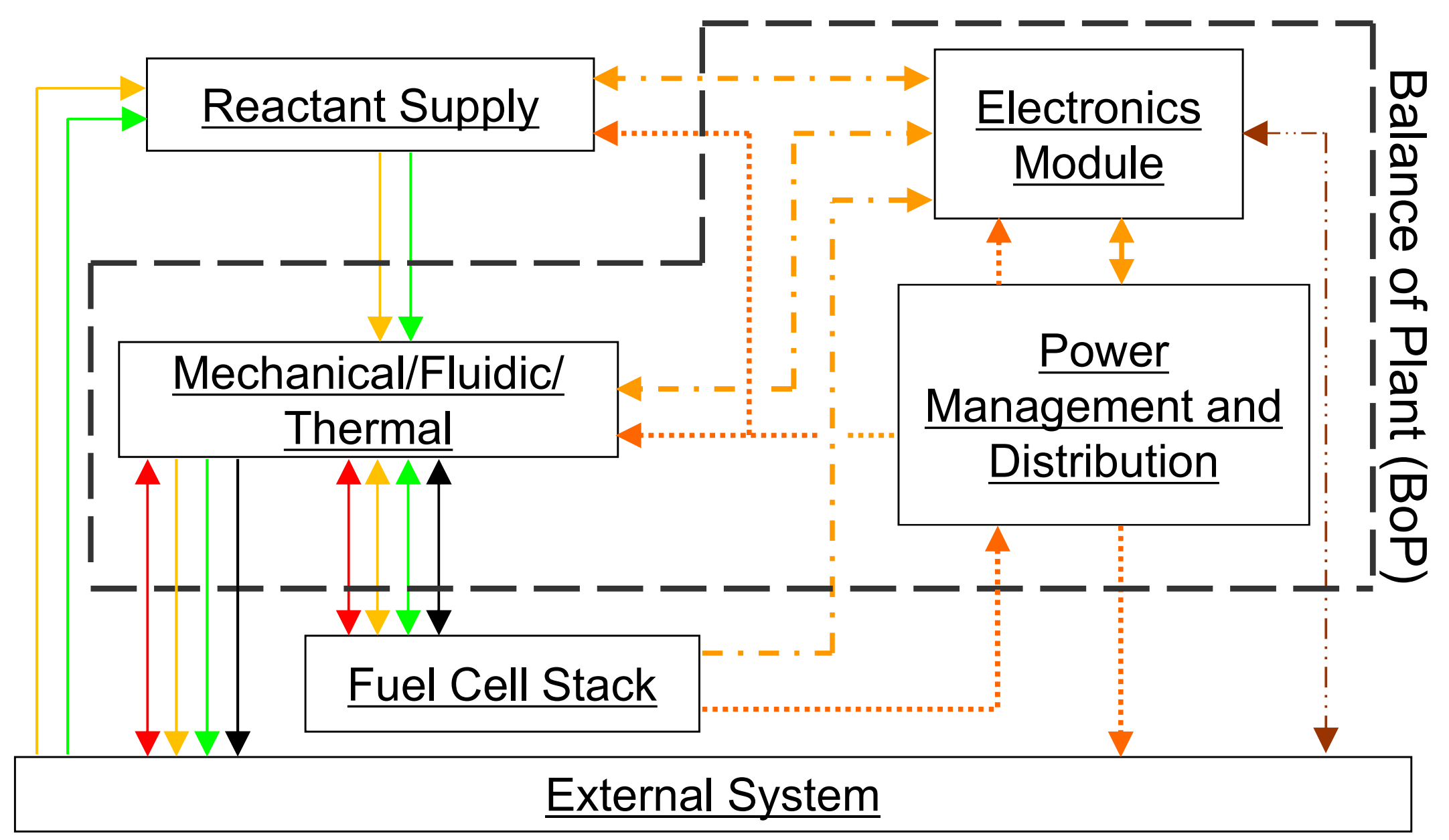

- Hydrogen Gas

- Oxygen Gas
- Heat

- Water/Coolant
- Communication Bus

- " - Sensor/Actuator

. . . . Power 


\section{AMPS 1kW APWR NFT PFC Power Module}

Advanced Modular Power Systems 1 Kilowatt Advanced Product Water Removal Non-Flow-Through Primary Fuel Cell Power Module
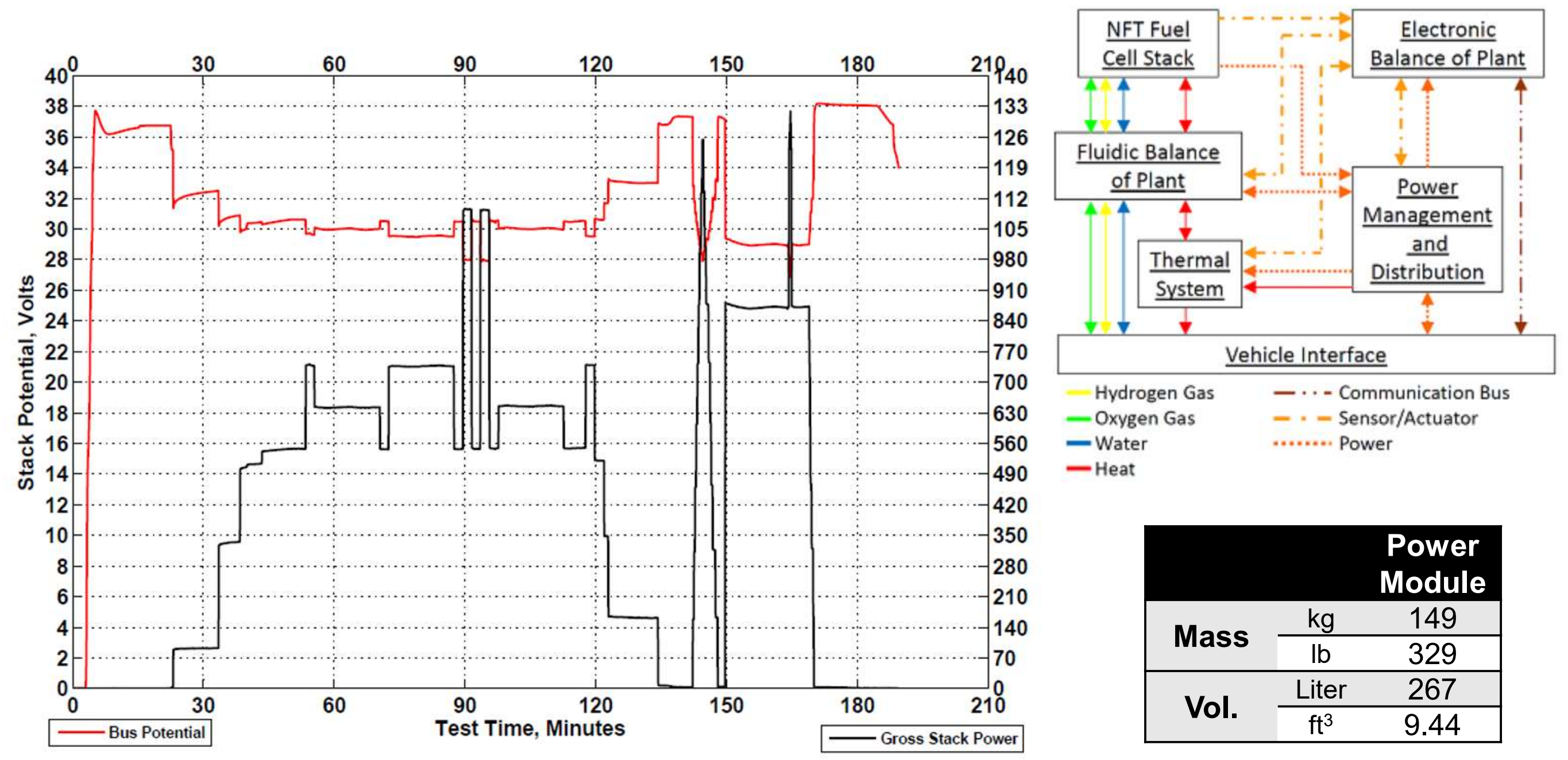


\section{The "Dunes"}

Outdoor test field for large scale vehicle demos and extended cross-slope testing

- $100 \mathrm{ft}$. X 80ft. graded area covered with 6 in. of sand

- Large hill with 3 sides of different slope angles: 10 , 15 , and 20 deg.

- Obstacle course consisting of boulders ( 2-3 ft.), moguls, and small hills

- Controls in place to limit vegetation and erosion and allow for drainage

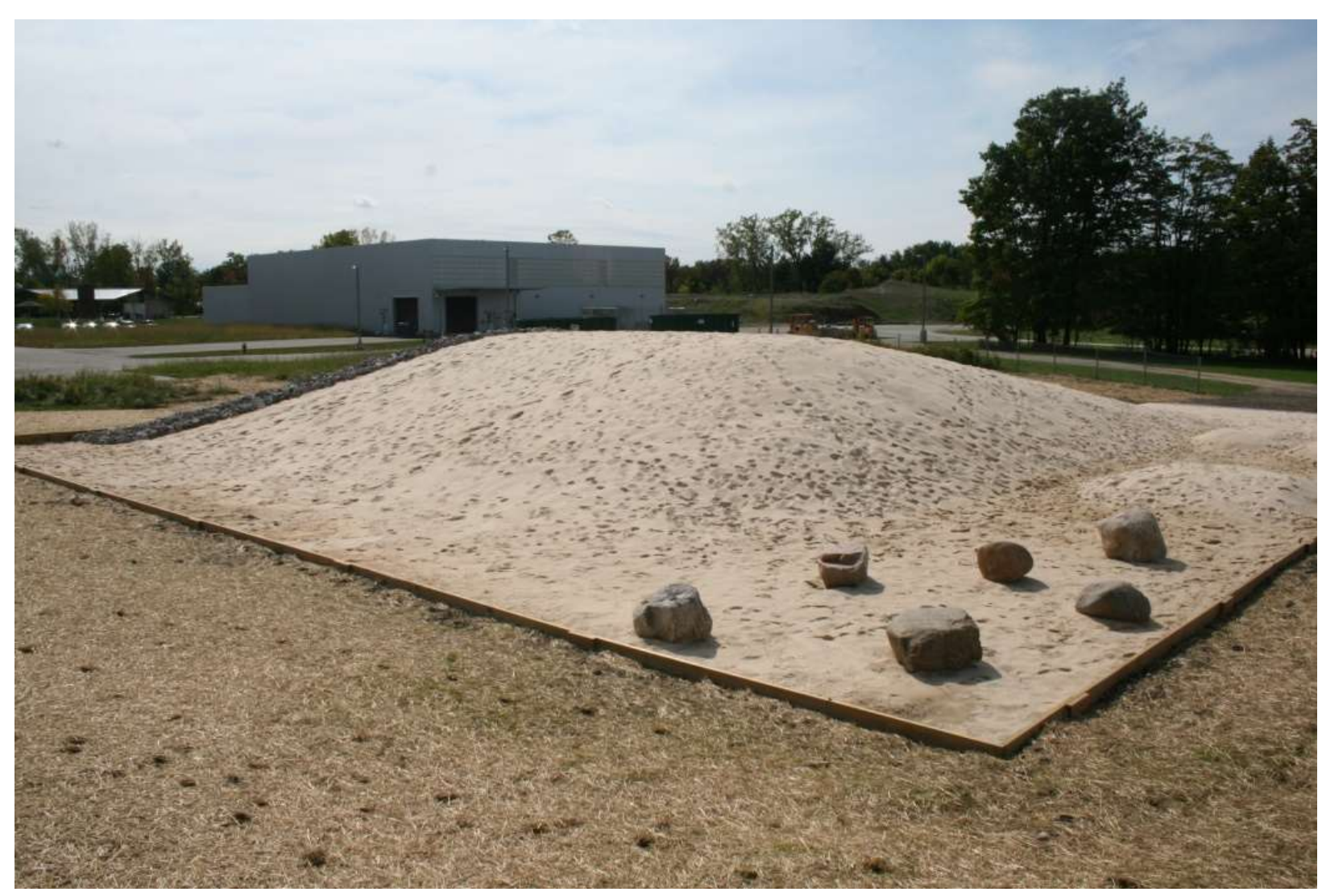

- Vehicle/trailer entrance 


\section{Scarab Rover}

\begin{tabular}{|l|l|}
\hline \multicolumn{2}{|c|}{ Scarab Specifications } \\
\hline Unloaded Mass & $300 \mathrm{~kg}$ \\
\hline Maximum Locomotion Speed & $5 \mathrm{~cm} / \mathrm{s}$ \\
\hline Wheelbase & $0.8-1.4 \mathrm{~m}$ \\
\hline Track width & $1.4 \mathrm{~m}$ \\
\hline CG height & $0.48-0.74 \mathrm{~m}$ \\
\hline Wheel diameter & $66-81 \mathrm{~cm}$ \\
\hline
\end{tabular}

\begin{tabular}{|l|c|c|}
\hline \multicolumn{3}{|c|}{ Scarab Power Demands, Watts } \\
\hline \multicolumn{1}{|c|}{ Motion } & $\begin{array}{c}\text { Motion } \\
\text { Power }\end{array}$ & $\begin{array}{c}\text { Total } \\
\text { Power }\end{array}$ \\
\hline Hotel Loads & 175 & 175 \\
\hline Stationary (Elevation Hold) & 6 & 181 \\
\hline Transit, Level hard ground & 85 & 255 \\
\hline Transit, Level loose sand & 120 & 305 \\
\hline Point Turn & 200 & 375 \\
\hline Elevating Body & 225 & 400 \\
\hline Peak Transient & 590 & 765 \\
\hline
\end{tabular}
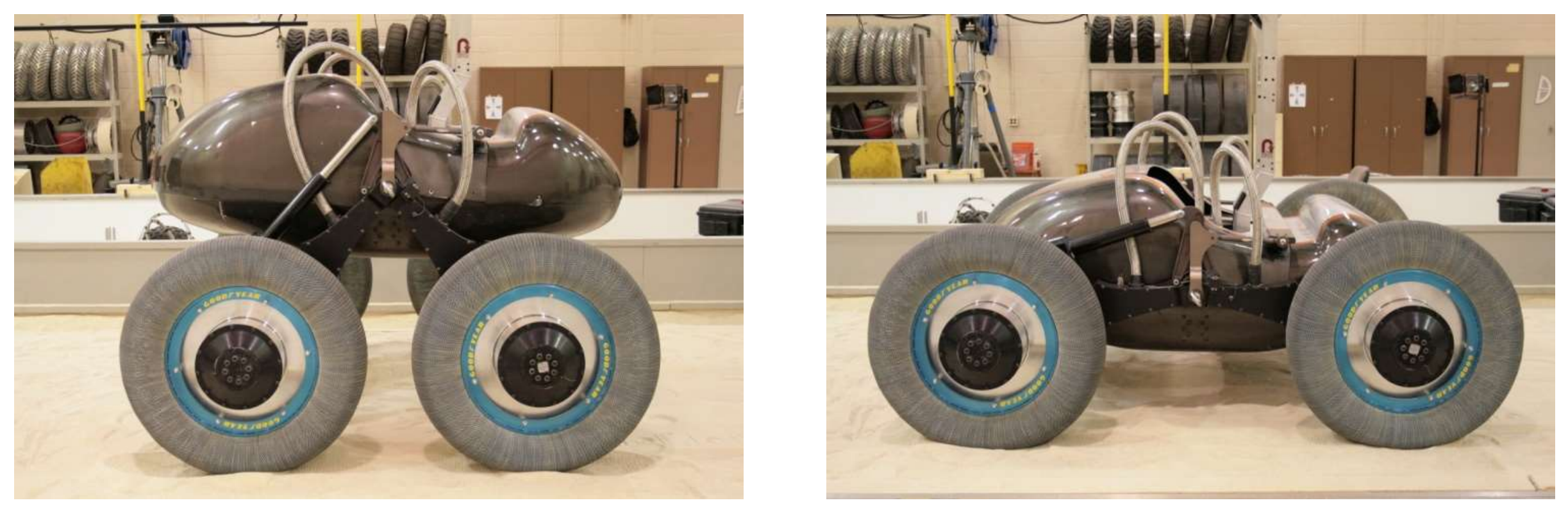


\section{Integrated Scarab Rover with Power Module}

\section{Advanced Modular Power Systems 1 Kilowatt Advanced Product}

Water Removal Non-Flow-Through Primary Fuel Cell Power Module

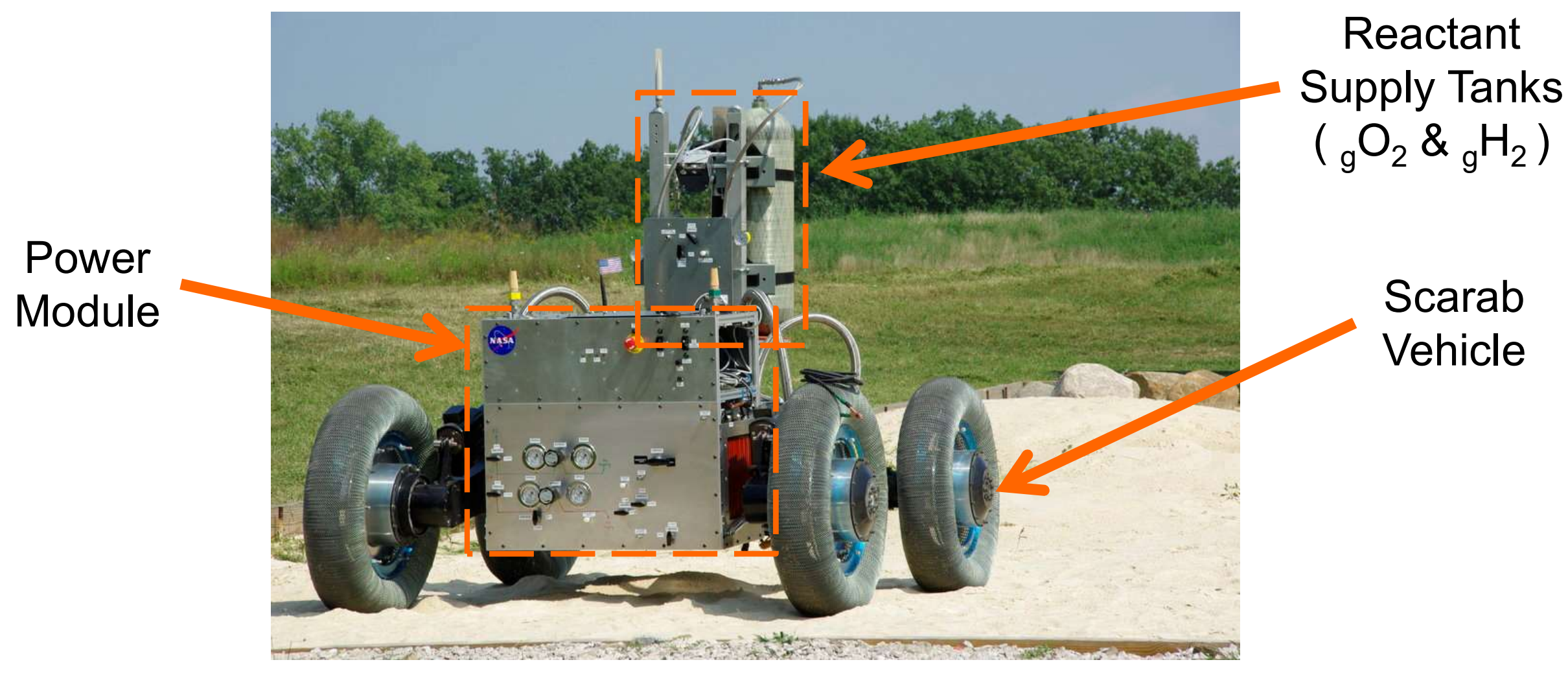

Integrated System Metrics

For Power Module with ${ }_{g} \mathrm{H}_{2} \&{ }_{g} \mathrm{O}_{2}$

Reactant Storage

\begin{tabular}{|ccc|}
\hline \multirow{2}{*}{ Specific Energy } & $\mathrm{W} \cdot \mathrm{hr} / \mathrm{kg}$ & 208 \\
\cline { 2 - 3 } & $\mathrm{kJ} / \mathrm{kg}$ & 749 \\
\hline Energy Density & $\mathrm{W} \cdot \mathrm{hr} / \mathrm{L}$ & 86 \\
\hline Power Density & $\mathrm{W} / \mathrm{L}$ & 2.26 \\
\hline
\end{tabular}

\begin{tabular}{|ccccc|} 
& & $\begin{array}{c}\text { Power } \\
\text { Module }\end{array}$ & $\begin{array}{c}\text { Reactant } \\
\text { System }\end{array}$ & $\begin{array}{c}\text { Total } \\
\text { System }\end{array}$ \\
\hline \multirow{2}{*}{ Mass } & $\mathrm{kg}$ & 149 & 34 & 183 \\
\cline { 2 - 5 } & $\mathrm{lb}$ & 329 & 75 & 404 \\
\hline \multirow{2}{*}{ Vol. } & $\mathrm{Liter}$ & 267 & 175 & 442 \\
\cline { 2 - 5 } & $\mathrm{ft}^{3}$ & 9.44 & 6.19 & 15.6 \\
\hline Stored & $\mathrm{kW} \cdot \mathrm{h}$ & $\mathrm{N} / \mathrm{A}$ & $\mathrm{N} / \mathrm{A}$ & 38 \\
\cline { 2 - 5 } Energy & $\mathrm{MJ}$ & $\mathrm{N} / \mathrm{A}$ & $\mathrm{N} / \mathrm{A}$ & 137 \\
\hline
\end{tabular}




\section{Demonstration Success Criteria}

\section{Success Criteria}

Power Module delivers at least $1 \mathrm{~kW}$ nominal power output within the voltage range of 24-36 VDC

SCARAB rover can start and idle with all power provided by the fuel cell power module (no power from additional external sources)

SCARAB rover can achieve forward, unidirectional motion on flat terrain at maximum speed with all power provided by the fuel cell power module

SCARAB rover can turn in place on flat terrain with all power provided by the fuel cell power module

SCARAB rover can utilize "inching" method of propulsion on flat terrain with all power provided by the fuel cell power module

SCARAB rover can climb a slope of at least 5 degrees with all power provided by the fuel cell power module

SCARAB rover can climb a slope up to 20 degrees with all power provided by the fuel cell power module

SCARAB rover can climb a slope at a crossing angle with all power provided by the fuel cell power module

SCARAB rover can navigate and climb small boulders and other obstacles at The Dunes test site with all power provided by the fuel cell power module
Priority

High

High

High

High

Medium

Medium

Low

Low

Low 


\section{Demonstration}

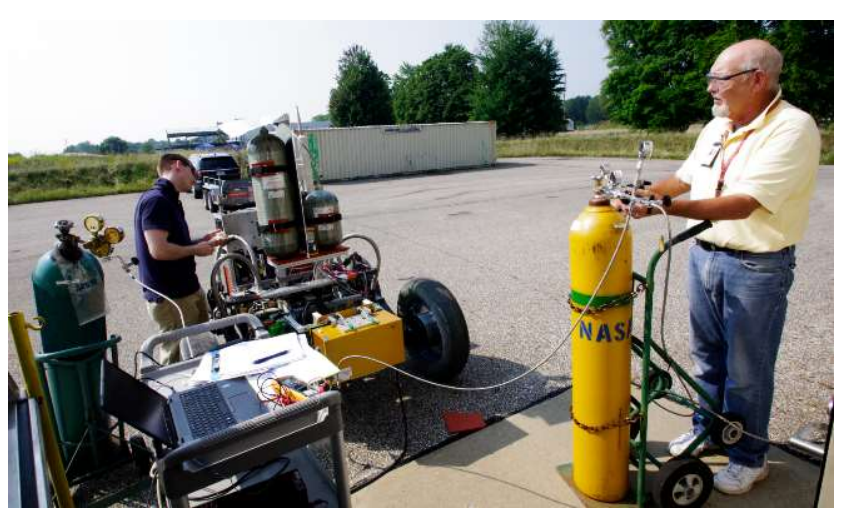

Charging the reactant gas tanks using the DTAU

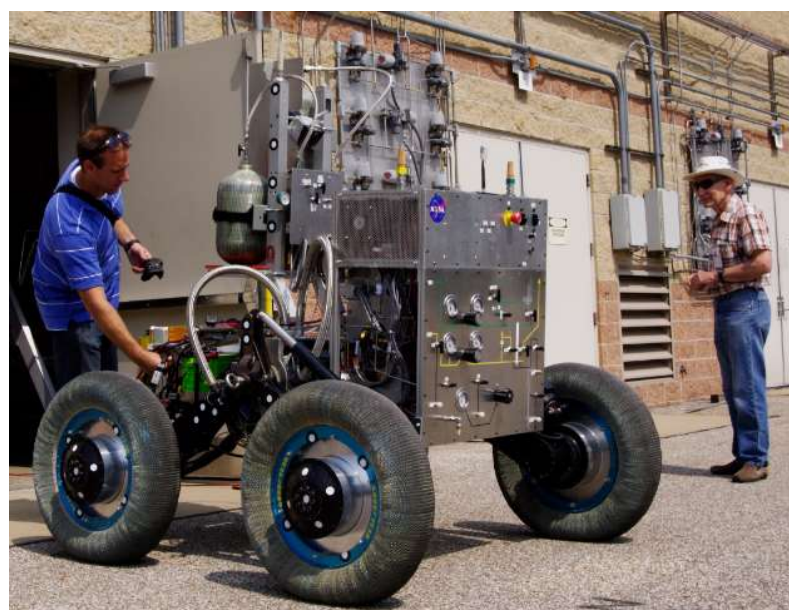

Integrated Vehicle Being Prepared for Checkout Testing on Level Surface

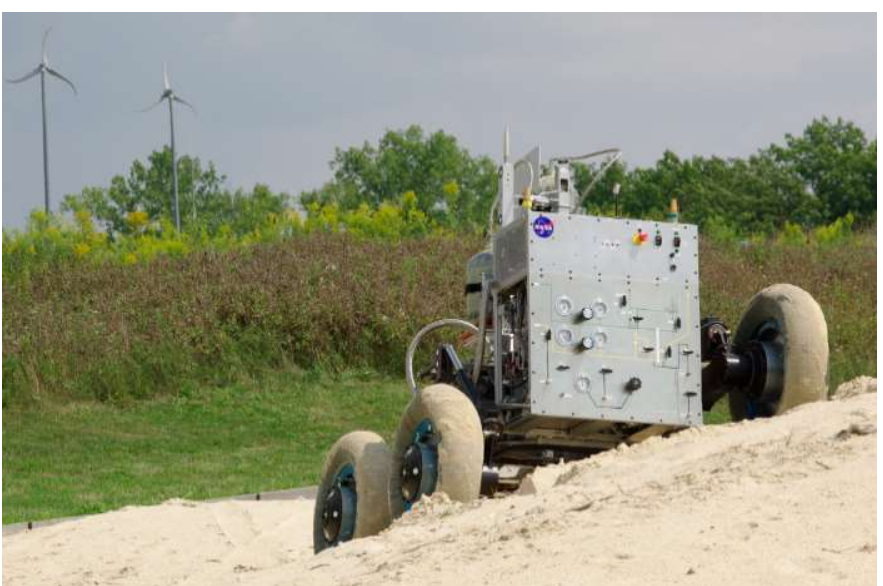

Integrated Vehicle Driving Across the $20^{\circ}$ Slope

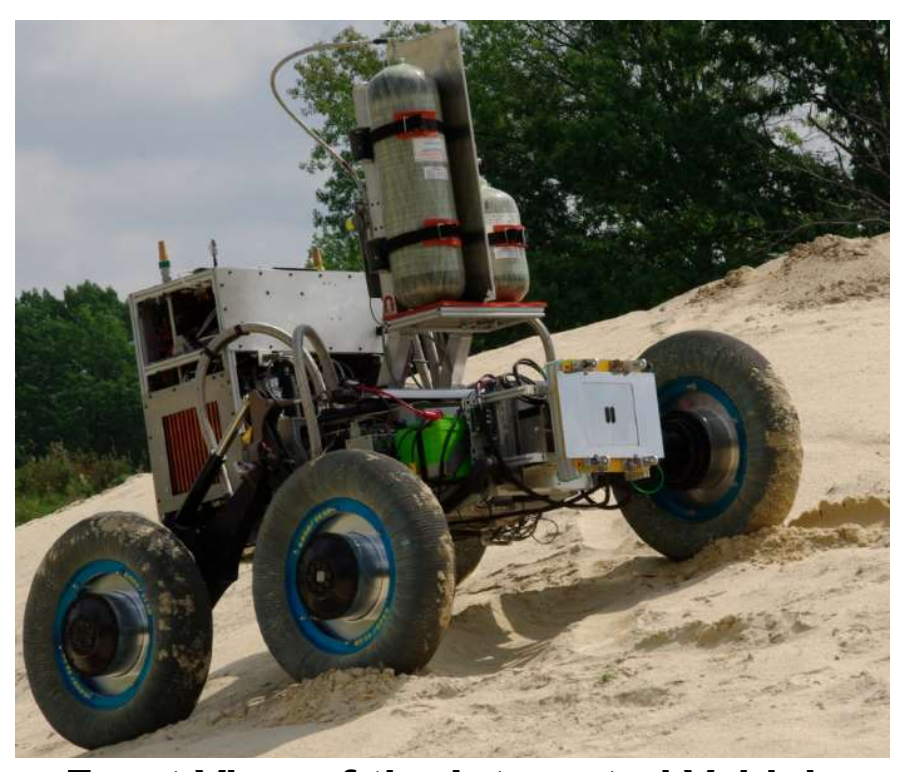

Front View of the Integrated Vehicle

\section{Success Criteria}

PM delivers $\geq 1 \mathrm{~kW}$ nominal power within 24-36 VDC

\section{Scarab, when powered by Fuel Cell Power module:}

- Starts and idles

- Moves forward, unidirectional motion on flat terrain at max speed

- Turns in place on flat terrain

- Utilizes "inching" method of propulsion on flat terrain

- Climbs a slope of at least 5 degrees

- Climbs a slope up to 20 degrees

- Climbs a slope at a crossing angle

- Climbs small boulders and other obstacles at The Dunes test site

\begin{tabular}{|c|c|c|}
\hline Success Criteria & Priority & \\
\hline PM delivers $\geq 1 \mathrm{~kW}$ nominal power within 24-36 VDC & High & $\sqrt{ }$ \\
\hline \multicolumn{3}{|l|}{ Scarab, when powered by Fuel Cell Power module: } \\
\hline - Starts and idles & High & $\sqrt{ }$ \\
\hline - Moves forward, unidirectional motion on flat terrain at max speed & High & $\sqrt{ }$ \\
\hline - Turns in place on flat terrain & High & $\sqrt{ }$ \\
\hline - Utilizes "inching" method of propulsion on flat terrain & Medium & $\sqrt{ }$ \\
\hline - Climbs a slope of at least 5 degrees & Medium & $\sqrt{ }$ \\
\hline - Climbs a slope up to 20 degrees & Low & $\sqrt{ }$ \\
\hline - Climbs a slope at a crossing angle & Low & $\sqrt{ }$ \\
\hline - Climbs small boulders and other obstacles at The Dunes test site & Low & $\sqrt{ }$ \\
\hline
\end{tabular}




\section{Review}

- Demonstration Scope

- Fundamental Technologies

- Advanced Product Water Removal (APWR) Non-Flow Through (NFT) Reactant Management

- Quad-Cell Voltage Monitoring Board (QCVMB)

- Dust Tolerant Automated Umbilical (DTAU)

- Power Module

- Rover

- Integrated Vehicle

- Demonstration

- Results

- Questions?

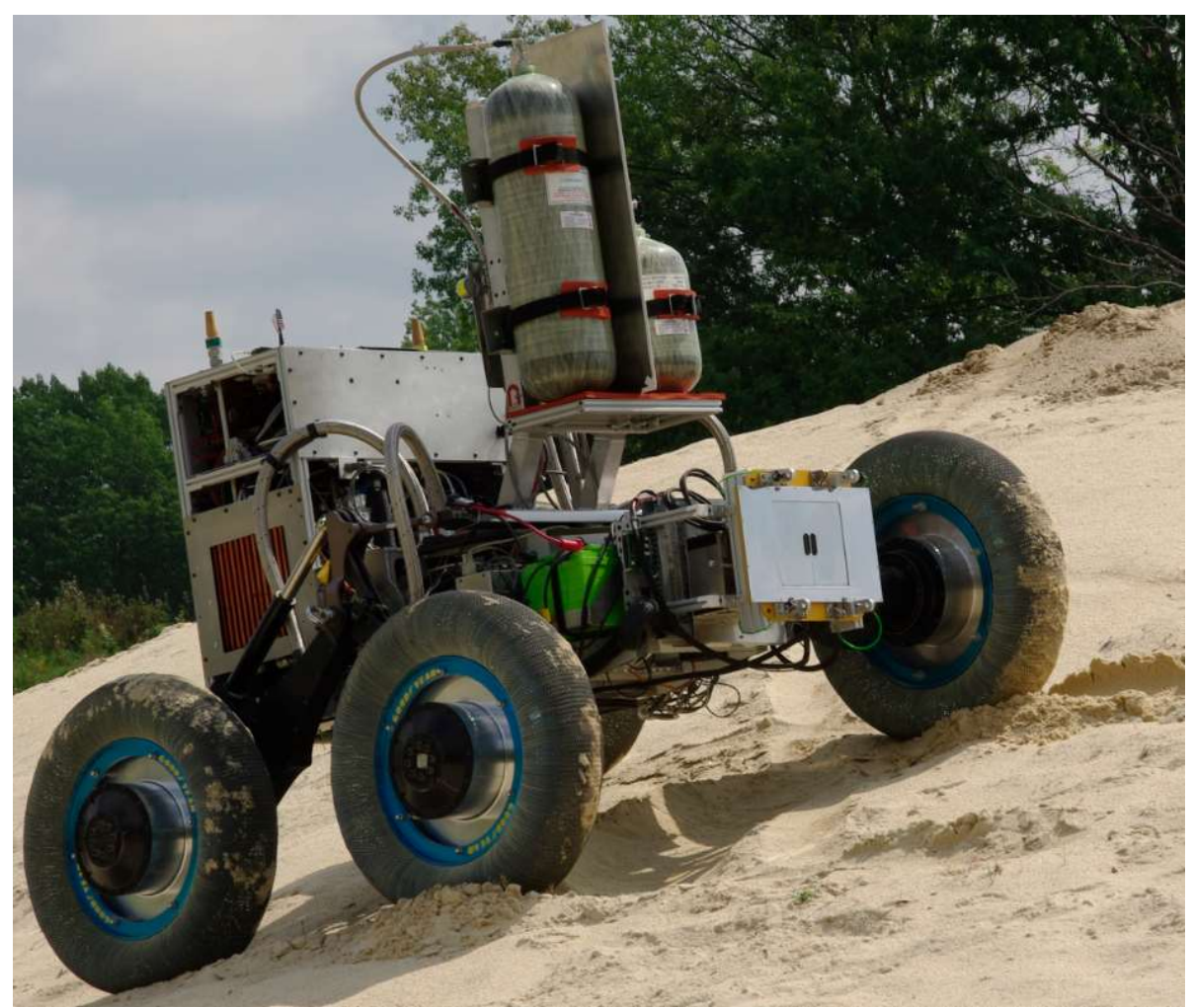




\section{Questions?}

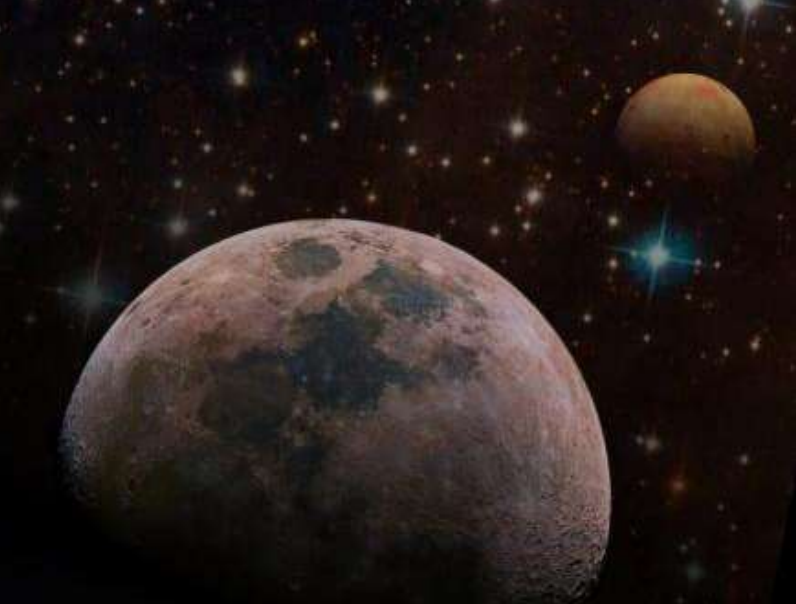




\section{BACK-UP SLIDES}


Advanced Modular Power Systems (AMPS)

Domain: Vehicle Systems

Lead Center: GRC

PM: Karin Bozak

Chief Technologist: Jim Soeder

$>$ AMPS will infuse new technology into power systems and components and prove their capabilities on exploration based ground demonstrations

$>$ AMPS will develop modular power units which, when combined with standardized interfaces can provide commonality across a variety of exploration vehicles

\section{Applications}

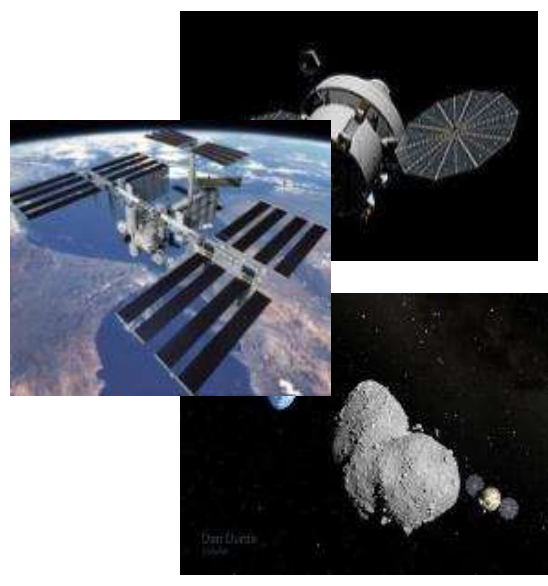

Exploration Missions

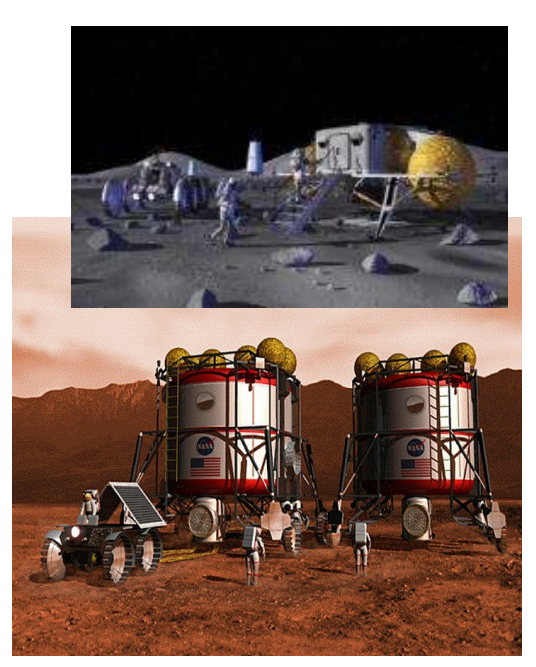

Planetary Outposts

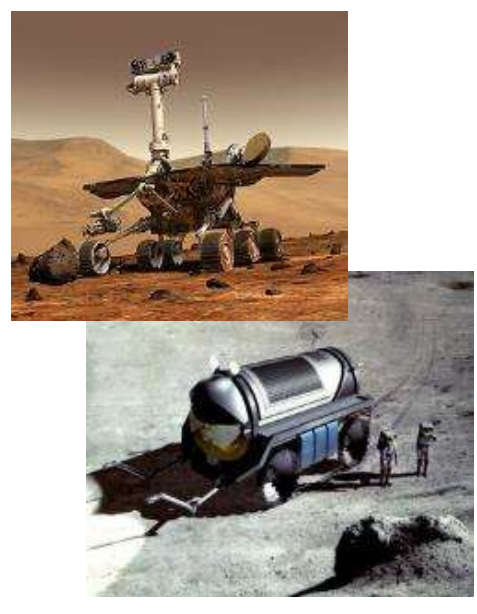

Mars / Lunar Rovers

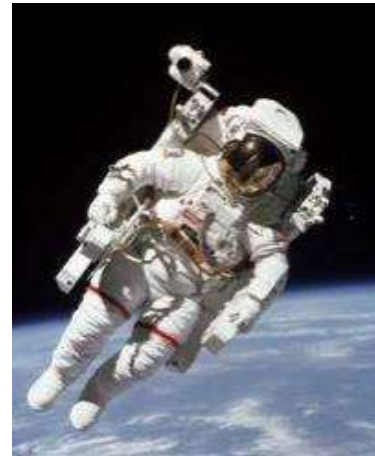

EVA Suits 


\section{NFT Operational Definitions}

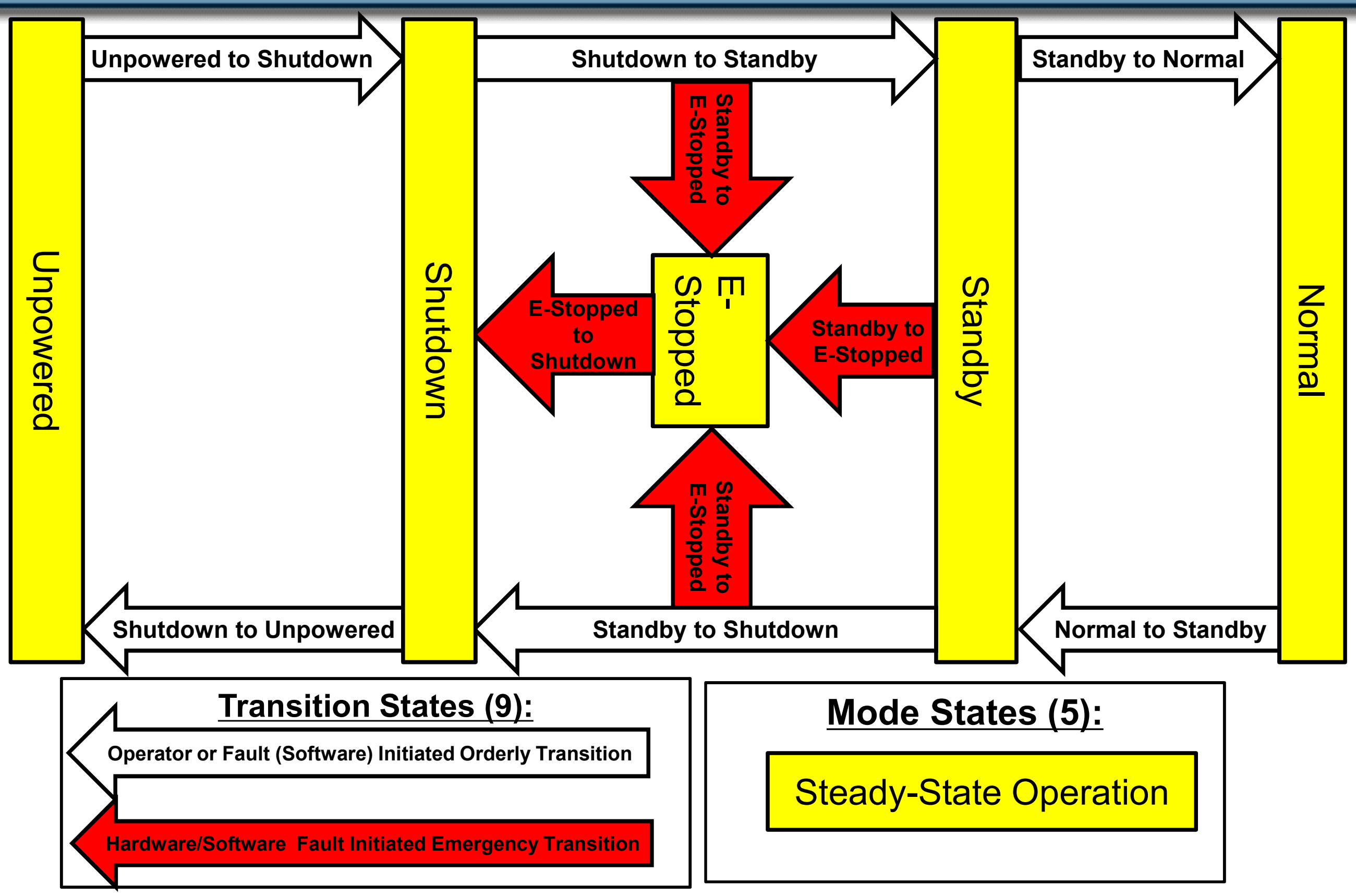




\section{Demonstration Test Matrix}

\begin{tabular}{|c|c|c|c|c|c|c|}
\hline Activity & Details & $\begin{array}{l}\text { Preparation/Equipment } \\
\text { Required }\end{array}$ & $\begin{array}{l}\text { \# of } \\
\text { Runs }\end{array}$ & $\begin{array}{c}\text { Time / } \\
\text { Run (min) }\end{array}$ & $\begin{array}{l}\text { Total Time } \\
\text { with setup } \\
\text { (min) }\end{array}$ & $\begin{array}{l}\text { Required } \\
\text { ? (Y/N) }\end{array}$ \\
\hline $\begin{array}{l}\text { Driving on flat } \\
\text { terrain }\end{array}$ & $\begin{array}{l}\text { Scarab driven forwards and } \\
\text { backwards. }\end{array}$ & Leveling soil & 3 & 20 & 90 & $Y$ \\
\hline Turn in place & $\begin{array}{l}\text { Turned in place on tilt-bed (more } \\
\text { space). Loads measured at various } \\
\text { angles. }\end{array}$ & None & 3 & 5 & 45 & $\mathrm{Y}$ \\
\hline Elevate body & $\begin{array}{l}\text { Scarab elevated from lowest to } \\
\text { highest positions. }\end{array}$ & None & 3 & 5 & 45 & $\mathrm{Y}$ \\
\hline Slope climbing & $\begin{array}{l}\text { Scarab driven up various slope angles } \\
(5,10,15,20 \text { deg). Angles may } \\
\text { change. Repeated if needed. }\end{array}$ & $\begin{array}{l}\text { S Loosening and leveling } \\
\text { soil. }\end{array}$ & 4 & 30 & 150 & $\mathrm{Y}$ \\
\hline $\begin{array}{l}\text { Cross-slope } \\
\text { climbing }\end{array}$ & $\begin{array}{l}\text { Repeat of slope climbing tests but } \\
\text { with Scarab leaning and driving } \\
\text { across the slope. Lower slope angles } \\
\text { not needed. }\end{array}$ & $\begin{array}{l}\text { Loosening and leveling } \\
\text { soil. }\end{array}$ & 3 & 30 & 120 & $\mathrm{~N}$ \\
\hline & $\begin{array}{l}\text { Scarab driven through obstacles } \\
\text { which consists of sand moguls, small }\end{array}$ & & & & & \\
\hline Obstacle course & $\begin{array}{l}\text { hill, rocks, and boulders. Tests can } \\
\text { be focused on specific obstacles of } \\
\text { interest. }\end{array}$ & None & 3 & 10 & 60 & $\mathrm{~N}$ \\
\hline Inching & $\begin{array}{l}\text { Repeat of driving of flat terrain but } \\
\text { using "inching" mode of travel. }\end{array}$ & Leveling soil & 3 & 20 & 90 & $\mathrm{~N}$ \\
\hline & & & \multicolumn{2}{|c|}{ Total Time $(\mathrm{hr})=$} & 10 & \\
\hline
\end{tabular}




\section{Demonstration Test Plan}

\begin{tabular}{|c|c|c|c|c|}
\hline Day & Location & Time of Day & Task & Time (min) \\
\hline \multirow{8}{*}{ Day 1} & \multirow{8}{*}{ The Dunes } & \multirow{4}{*}{ Morning } & Start up Scarab and fuel cell & 45 \\
\hline & & & Check-out system & 60 \\
\hline & & & Test out stationary/hotel loads & 15 \\
\hline & & & Flat terrain tests & 120 \\
\hline & & \multirow{4}{*}{ Afternoon } & Turn in place tests & 45 \\
\hline & & & Elevate body tests & 45 \\
\hline & & & Inching Tests & 90 \\
\hline & & & Shut down Scarab and fuel cell & 45 \\
\hline \multirow{7}{*}{ Day 2} & \multirow{6}{*}{ The Dunes } & \multirow{4}{*}{ Morning } & Start up Scarab and fuel cell & 45 \\
\hline & & & Check-out system / verify hotel loads & 15 \\
\hline & & & Repeat turn in place, inching tests at minimal level & 60 \\
\hline & & & Start slope-climbing tests (up to $20 \mathrm{deg}$ ) & 75 \\
\hline & & \multirow[b]{2}{*}{ Afternoon } & Continue slope-climbing tests & 75 \\
\hline & & & Cross-slope climbing tests (up to $20 \mathrm{deg}$ ) & 120 \\
\hline & & & & 25 \\
\hline \multirow{9}{*}{ Day 4 - "Media Day" } & \multirow{9}{*}{$\begin{array}{l}\text { The Dunes } \\
\text { (weather } \\
\text { permitting) }\end{array}$} & \multirow{6}{*}{ Morning } & Transfer Scarab and equipment to Dunes & 60 \\
\hline & & & Start up Scarab and fuel cell & 45 \\
\hline & & & Check-out system / verify hotel loads & 15 \\
\hline & & & Slope-climbing tests/demos & 90 \\
\hline & & & Cross-slope climbing tests $(10,15$, and 20 deg) & 60 \\
\hline & & & Demonstrate turning/elevating/inching & 60 \\
\hline & & \multirow[t]{3}{*}{ Afternoon } & Obstacle course demos & 60 \\
\hline & & & Shut down Scarab and fuel cell & 45 \\
\hline & & & Transfer Scarab and equipment to bldg. 334 & 45 \\
\hline
\end{tabular}

Total Time $(\mathrm{hr})=8$ 